PACS: 85.30.Tv

\title{
Accurate numerical modelling the GaAs MESFET current-voltage characteristics
}

\author{
N. Merabtine ${ }^{1}$, S. Khemissi ${ }^{2}$, M. Zaabat ${ }^{3}$, M. Belgat ${ }^{4}$, C. Kenzai ${ }^{4}$ \\ 1 Laboratoire Electromagnetisme et Telecommunication, Electronics Department, Faculty of Engineering, \\ University, Mentouri, Constantine, Algeria \\ E-mail:na_merabtine@hotmail.com \\ 2 Departement de Seti, Faculté de technologie Université de khenchla, Algeria \\ E-mail: saadekhemissi@yahoo.fr \\ 3 Physics department, University of Oum-El-Bouaghi, Algeria \\ E-mail: Zaabat@hotmail.com \\ 4 Laboratoire des Couches Minces et Interfaces, Département de physique Faculté des Sciences, Université \\ Mentouri de Constantine \\ E-mail: musbelgat@yahoo.fr
}

\begin{abstract}
In this paper, we present a computing model of the current-voltage $(I-V)$ characteristics of a gallium arsenide Schottky barrier field effect transistor called GaAs MESFET. This physical model is based on the two-dimensional analysis of the Poisson equation in the active region under the gate. In this frame, we elaborated a simulation software based on analysis of expressions that we have previously set up [1-3], the obtained theoretical results are discussed and compared to the experimental ones.
\end{abstract}

Keywords: gallium arsenide, field effect transistor, Poisson equation.

Manuscript received 11.08.04; accepted for publication 16.12.04.

\section{Introduction}

Essentially, the Gallium Arsenide Field Effect Transistors (GaAs FET) are high-speed and highfrequency devices. In this paper, we propose a simulation of the current-voltage $(I-V)$ characteristics of a short-gate GaAs MESFETs. Computer-aided analysis is a useful technology for studying physical phenomena in semiconductors. Here, we presented two-dimensional simulation of GaAs MESFET considering the physical model. Two-dimensional solution of the Poisson equation has been obtained taking into account structure study results.

\section{Physical model}

Fig. 1 shows a normal planar GaAs MESFET simulated in this paper. Two-dimensional physical model is used to solve the Poisson equation. This equation known in semiconductor physics is used in all the models to explain the different physical phenomena specific to the GaAs MESFET [4-6].

But the main problem for these models lies in the coupling of partial and nonlinear differential equations, which require to be simultaneously solved. The difficulty of putting down a valid hypothesis for the limit conditions at the free interface requires the resort to approximations the negligence of a certain number of terms that act negatively on the model exactness [4-6].

In this paper, we present an analytical model that combines the description of physical phenomena and simplicity of solving the respective mathematical equation.

\section{Determination of the two-dimensional voltage in the active region}

The two-dimensional solution of differential equations using the Green method gives a distribution of the electric field under the region of the space charge area (SCA). The general Poisson equation is given by

$\Delta V_{c}(x, y)=\frac{\partial^{2} V_{c}}{\partial x^{2}}+\frac{\partial^{2} V_{c}}{\partial y^{2}}=\frac{-\rho(x, y)}{\varepsilon}$.

To calculate the voltage under the gate, the SCA is divided into two main regions shown in Fig. 1.

- The region (1) is directly under the gate, it is considered as a region controlled by the gate. We use the uni-dimensional approximation to calculate the composant of the relation of the voltage $V_{q}(x, y)$ specific to this region.

- The region (2) outside the first region is considered as uncontrolled by the gate. The two-dimensional voltage of the channel under the gate is given as follows: 


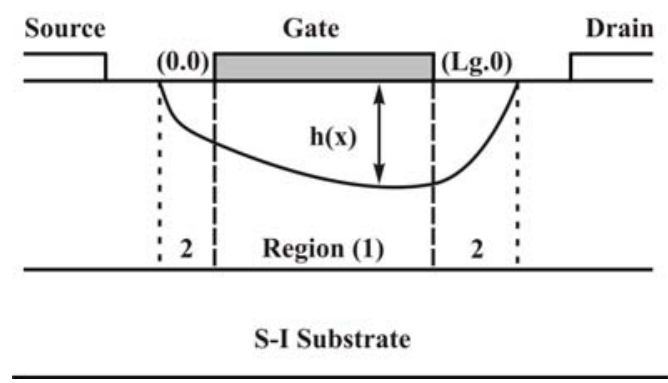

Fig. 1. Depletion regions controlled (1) and uncontrolled (2) by the gate.

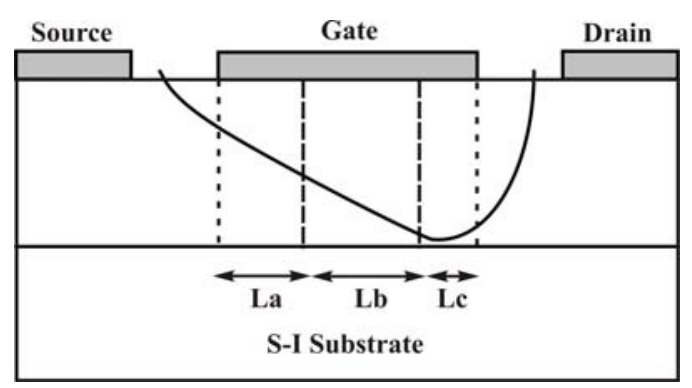

Fig. 2. Active area distribution according to the electric field variation.

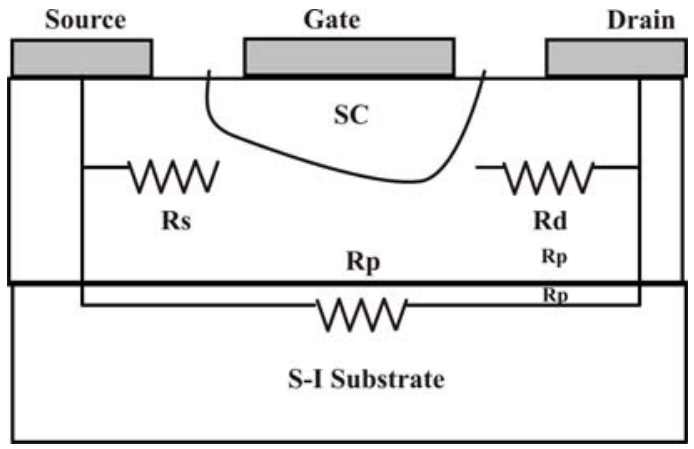

Fig. 3. Parasitic resistance of GaAs MESFET.

$$
V_{c}(x, y)=V_{q}(x, y)+V_{l}(x, y),
$$

where

$V_{q}(x, y)=\int_{0}^{y} \frac{e N_{d}(x, y)}{\varepsilon} y d y+y \int_{y}^{h(x)} \frac{e N_{d}(x, y)}{\varepsilon} d y+V_{b i}+V_{g}$,

and

$V_{l}(x, y)=\left[A_{1}^{s} \frac{\sinh \left(k_{1}(L-x)\right)}{\sinh \left(k_{1} L\right)}+A_{1}^{d} \frac{\sinh \left(k_{1} x\right)}{\sinh \left(k_{1} L\right)}\right] \sin \left(k_{1} y\right)$

with

$A_{1}^{s}=\frac{2}{a} \int_{0}^{a}\left[V_{c}(0, y)-V_{q}(0, y)\right] \sin \left(k_{1} y\right) d y$ and

$A_{1}^{d}=\frac{2}{a} \int_{0}^{a}\left[V_{c}(L, y)-V_{q}(L, y)\right] \sin \left(k_{1} y\right) d y$.

$A_{1}^{d}$ and $A_{1}^{s}$ are the Fourier coefficients for the gate supplementary voltage for the drain and source sides, respectively [5],

and $k_{1}=\frac{\pi}{2 a}$.

If considering (3) and (4), the total voltage expression becomes

$V_{c}(x, y)=\int_{0}^{h(x)} \frac{e N_{d}(x, y)}{\varepsilon} y d y+V_{l}(x, y)-V_{g}+V_{b i}$.

\section{Current-voltage characteristics}

To calculate the drain current expression as a function of the drain voltage for different values of the gate voltage, we use the following hypothesis:

- we neglect the current in the $Y$-axis, this approximation is valid for the short-gate components;

- $\quad$ we suppose the electrons mobility constant;

- $\quad$ we derived the channel in three regions according to the electric field value (Fig. 2) [6].

\section{Determination of the current general equation}

To calculate the drain current general equation, we used the uni-dimensional approximation to simplify the mathematical expressions. We also use the following expressions:

$\vec{J}_{x}=-e \mu_{n} N_{d}(y) E_{x}=-e \mu_{n} N_{d} \frac{d V}{d x}$,

the drain current expression is given by

$I_{d}=-\int_{(s)} J_{x} d s=-Z \int_{h(x)}^{a} J_{x} d y$,

using single integrals, the current expression is obtained by relation

$I_{d}=\frac{\left(e N_{d}\right)^{2} Z \mu_{n}}{\varepsilon L}\left[\frac{a}{2}\left(h_{d}^{2}-h_{s}^{2}\right)-\frac{1}{3}\left(h_{d}^{3}-h_{s}^{3}\right)\right]$

where

$h_{s}=\left[\frac{2 \varepsilon}{e N_{d}}\left(V_{b i}-V_{g}\right)\right]^{1 / 2}$,
$h_{d}=\left[\frac{2 \varepsilon}{e N_{d}}\left(V_{d}+V_{b i}-V_{g}\right)\right]^{1 / 2}$

are the widths of the space charge area (SCA) respectively source side and drain side. 

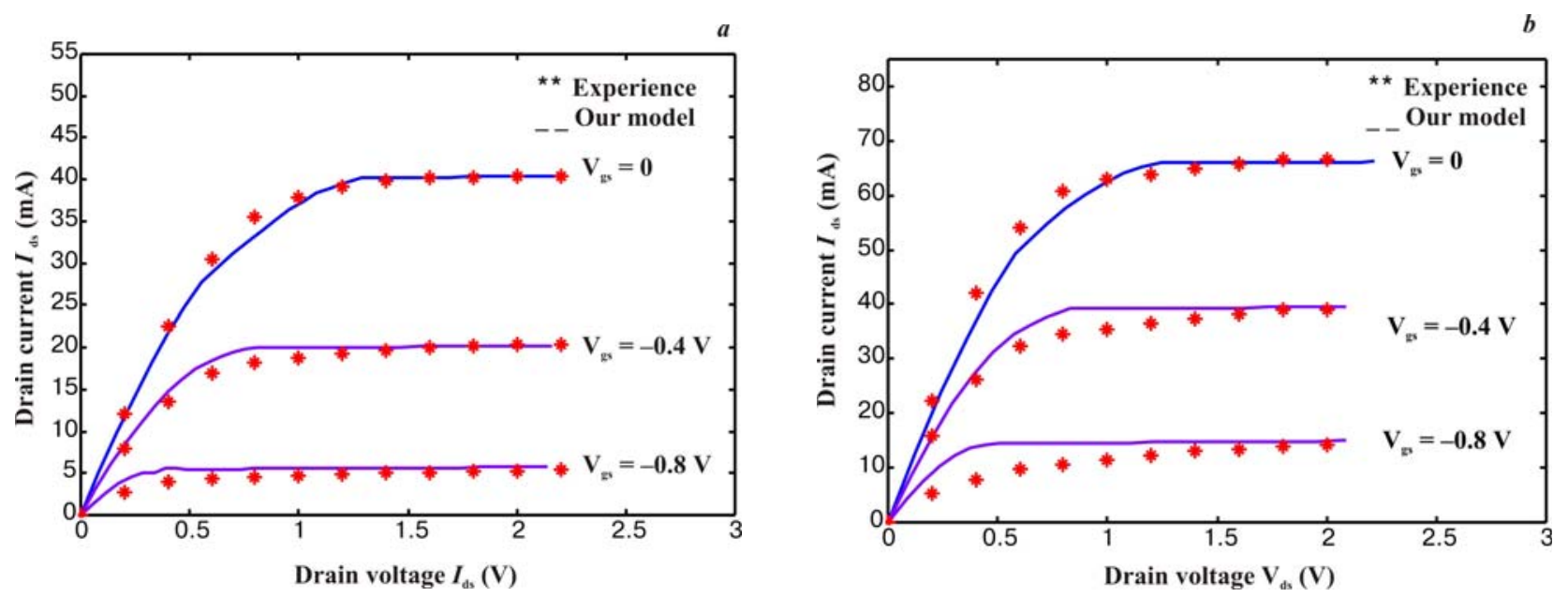

Fig. 4. Comparison of the $I-V$ characteristics measured and calculated by the simulation for MESFETs 1 (a) and 2 (b).
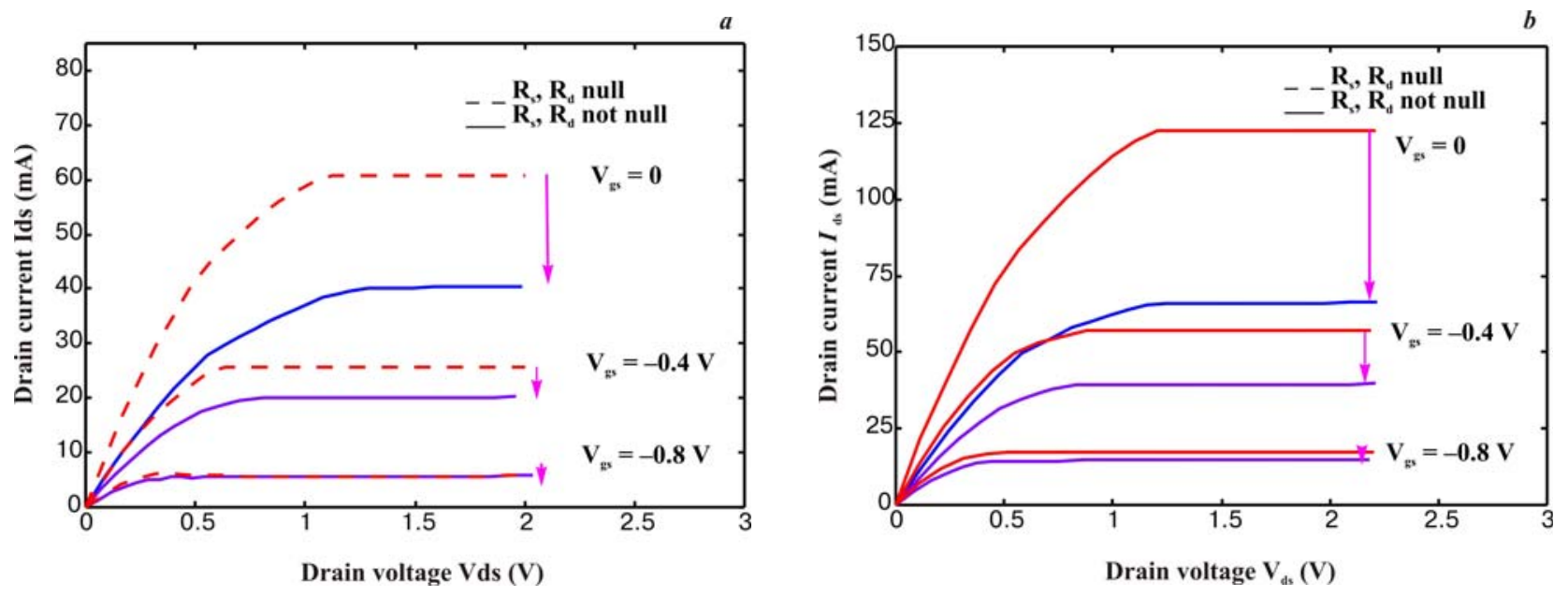

Fig. 5. Effect of the parasitic resistances on the $I$ - $V$ characteristics for MESFETs 1 (a) and 2 (b).
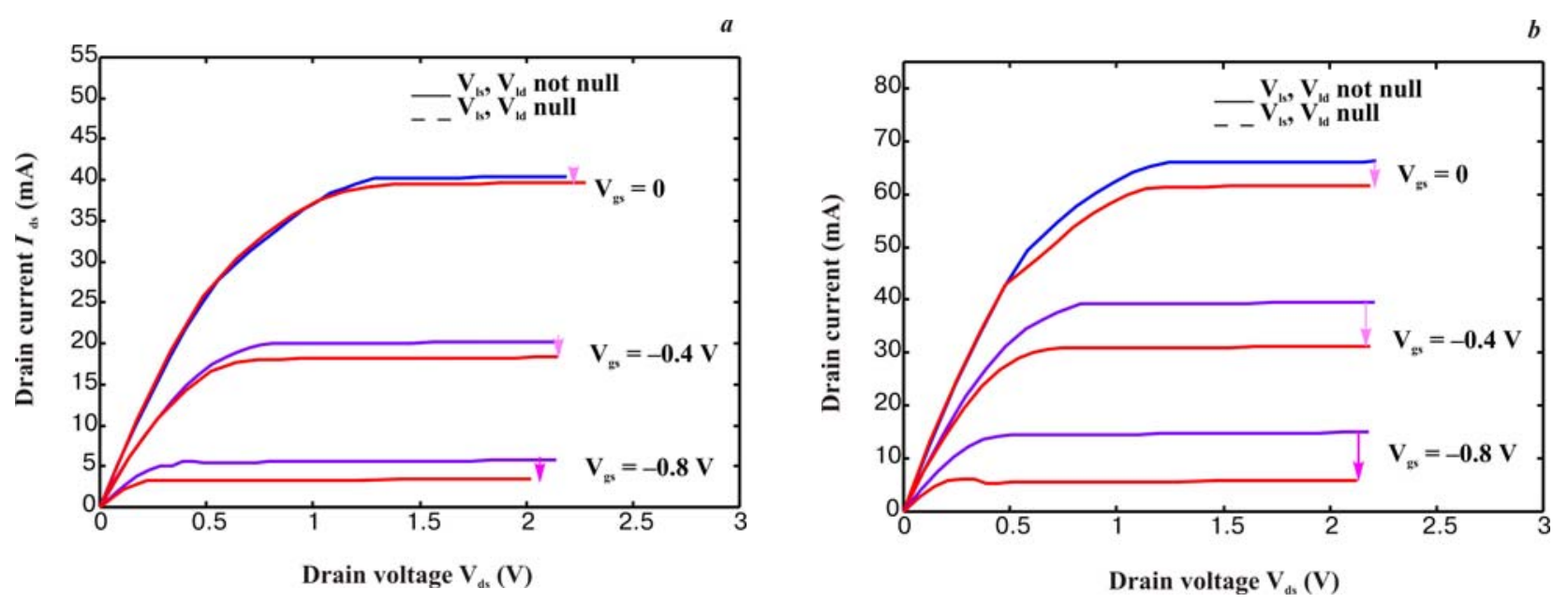

Fig. 6. Effect of the side voltages $V_{l s}$ and $V_{l d}$ on the $I-V$ characteristics for MESFETs 1 (a) and 2 (b). 
Defining the pinch-off current $I_{p}$ is given by

$$
I_{p}=\frac{\left(e N_{d}\right)^{3} Z \mu a^{3}}{2 \varepsilon L}
$$

and the pinch-off voltage $V_{p}$ is given by

$V_{p}=\frac{e N_{d}}{2 \varepsilon} a^{2}$.

The general equation expression $I_{P}$ in the channel becomes

$$
I_{d}=I_{p}\left[\frac{V_{d}}{V_{p}}-\frac{2}{3}\left(\frac{V_{d}+V_{b i}-V_{g}}{V_{p}}\right)^{3 / 2}+\frac{2}{3}\left(\frac{V_{b i}-V_{g}}{V_{p}}\right)^{3 / 2}\right] .
$$

\section{Effect of the mobility law}

The hypothesis of the constant mobility and the independency of the electric field in the $n$-type GaAs can not convey the physical phenomena. The analytical expression of the mobility variations with the electric field used by us is a simplified relation [7, 8] given as follows:

- for the weak electric field where $E<E_{0}$ $\mu=\mu_{0}$;

- $\quad$ for the high electric field beyond $E_{0}\left(E>E_{0}\right)$

$$
\mu=\frac{\mu_{0}}{\left[1+\left(\frac{E-E_{0}}{E_{s}}\right)^{2}\right]^{1 / 2}} .
$$

This mobility law allows to obtain the different expressions of the drain current in different operation regimes.

$I_{d}\left(V_{d}, V_{g}\right)$ characteristics of the GaAs MESFET corresponding to different operation regimes obey the equations considered below.

Table 1.

\begin{tabular}{|c|c|c|c|}
\hline \hline Transistor & $L, \mu \mathrm{m}$ & $A, \mu \mathrm{m}$ & $Z, \mu \mathrm{m}$ \\
\hline MESFET 1 & 1 & 0.153 & 300 \\
\hline MESFET 2 & 0.5 & 0.1435 & 300 \\
\hline \hline Transistor & $\mu_{0}, \mathrm{~m}^{2} / \mathrm{V} \cdot \mathrm{s}$ & \multicolumn{2}{|c|}{$N_{d}, 10^{23} \mathrm{~m}^{-3}$} \\
\hline MESFET 1 & 0.4000 & \multicolumn{2}{|c|}{1.17} \\
\hline MESFET 2 & 0.4000 & \multicolumn{2}{|c|}{1.31} \\
\hline \hline Transistor & $V_{s}, \mathrm{~m} / \mathrm{s}$ & $V_{b i}, \mathrm{~V}$ & $V_{p}, \mathrm{~V}$ \\
\hline MESFET 1 & $3.6 \cdot 10^{3}$ & 0.85 & 1.93 \\
\hline MESFET 2 & $7.3 \cdot 10^{3}$ & 0.85 & 1.93 \\
\hline
\end{tabular}

Table 2.

\begin{tabular}{|c|c|c|c|c|}
\hline Transistor & $a_{1}$ & $b_{1}$ & $c_{1}$ & $V_{l} / V_{p}$ \\
\hline MESFET 1 & -0.10 & 0.10 & 0.05 & 0.01 \\
\hline MESFET 2 & -0.14 & 0.10 & 0.04 & 0.01 \\
\hline
\end{tabular}

\subsection{Linear regime}

This regime exists as far as $L_{a}$ occupies all the channel, it corresponds to the weak field area where the mobility is equal to $\mu_{0}$.

The drain current expression in this regime is given as

$$
I_{d}=I_{p l}\left[\frac{V_{d}}{V_{p}}-\frac{2}{3}\left(\frac{2}{3} \frac{V_{d}+V_{b i}-V_{g}}{V_{p}}\right)^{3 / 2}+\frac{2}{3}\left(\frac{V_{b i}-V_{g}}{V_{p}}\right)^{3 / 2}\right]
$$

where

$I_{p l}=\frac{e^{2} N_{d}^{2} Z \mu_{0} a^{3}}{2 \varepsilon L_{a}}$.

\subsection{Pinch-off regime}

As the drain voltage increases, the electric field in the channel increases beyond $E_{0}$. The channel under the gate can be then represented by two regions.

One region of the length $L_{a}$ in which the field is inferior to $E_{0}$ and the electron mobility is constant and given by $\mu=\mu_{0}$. Another region of the length $L_{b}\left(L=L_{a}+L_{b}\right)$ in which the field is superior to the field $E_{0}$ but inferior to the field $E_{m}$, and the electron mobility is given by the expression (15b).

First region: for $E<E_{0}$ and $0<x<L_{a}$

$$
L_{a}=\frac{I_{p l} L}{I_{d}}\left[\frac{V_{d a}}{V_{p}}-\frac{2}{3}\left(\frac{V_{d a}+V_{b i}-V_{g}}{V_{p}}\right)^{3 / 2}-\frac{2}{3}\left(\frac{V_{b i}-V_{g}}{V_{p}}\right)^{3 / 2}\right] .
$$

Second region: for $E_{0}<E<E_{m}$ and $L_{a}<x<L$

$L_{b}=\frac{I_{p s} L}{I_{d}}\left[\frac{V_{d}-V_{d a}}{V_{p}}-\frac{2}{3}\left(\frac{V_{d}+V_{b i}-V_{g}}{V_{p}}\right)^{3 / 2}+\left(\frac{V_{d a}+V_{b i}-V_{g}}{V_{p}}\right)^{3 / 2}\right]$

where

$$
I_{p s}=\frac{I_{p}}{\left[1+\left(\frac{E-E_{0}}{E_{s}}\right)^{2}\right]^{1 / 2}} .
$$

\subsection{Saturation regime}

In this case, the channel under the gate is divided into three regions $L_{a}, L_{b}$, and $L_{c}$ where $L=L_{a}+L_{b}+L_{c}$.

$L_{a}=\frac{I_{p l} L}{I_{d}}\left[\frac{V_{d a}}{V_{p}}-\frac{2}{3}\left(\frac{V_{d a}+V_{b i}-V_{g}}{V_{p}}\right)^{3 / 2}-\frac{2}{3}\left(\frac{V_{b i}-V_{g}}{V_{p}}\right)^{3 / 2}\right]$, 


$$
\begin{aligned}
& L_{b}=\frac{I_{p s} L}{I_{d}}\left[\frac{V_{d m}-V_{d a}}{V_{p}}-\frac{2}{3}\left(\frac{V_{d m}+V_{b i}-V_{g}}{V_{p}}\right)^{3 / 2}+\frac{2}{3}\left(\frac{V_{d a}+V_{b i}-V_{g}}{V_{p}}\right)^{3 / 2}\right], \\
& L_{c}=\frac{I_{p s} L}{I_{d}}\left[\frac{V_{d}-V_{d m}}{V_{p}}-\frac{2}{3}\left(\frac{V_{d}+V_{b i}-V_{g}}{V_{p}}\right)^{3 / 2}+\frac{2}{3}\left(\frac{V_{d m}+V_{b i}-V_{g}}{V_{p}}\right)^{3 / 2}\right]
\end{aligned}
$$

where $V_{d a}$ and $V_{d m}$ are successively maximum and pinchoff voltages for linear regimes.

\section{Effect of the voltage $V_{l}(x, y)$}

The effect of the voltage $V_{l}(x, y)$ is taken into consideration in the following expressions of the drain and gate voltages:

$V_{d} \rightarrow V_{d}+V_{l d}$ and $V_{g} \rightarrow V_{g}+V_{l s}$

where

$V_{l s}=V_{l}\left(0, h_{s}\right)=A_{1}^{s} \sin \left(\frac{h_{s} \pi}{2 a}\right)$,

$V_{l d}=V_{l}\left(L, h_{d}\right)=A_{1}^{d} \sin \left(\frac{h_{d} \pi}{2 a}\right)$.

The coefficient expressions $A_{1}^{s}$ and $A_{1}^{d}$ are very complex [6], they are essentially related to the polarization voltages $V_{d}$ and $V_{g}$ and to the voltages $V_{b i}$ and $V_{p}$ :

$$
\begin{aligned}
& A_{1}^{S}=V_{p}\left[a_{1}+b_{1}\left(\frac{V_{b i}-V_{g}-V_{l}}{V_{p}}-c_{1}\right)^{1 / 2}\right], \\
& A_{1}^{d}=V_{p}\left[a_{1}+b_{1}\left(\frac{V_{d}+V_{b i}-V_{g}-V_{l}}{V_{p}}-c_{1}\right)^{1 / 2}\right] .
\end{aligned}
$$

For uniform doping, the coefficients $a_{1}, b_{1}, c_{1}$, and $V_{l}$ are constants.

\section{Effect of parasitic elements}

The characteristics that we have presented are those concerning internal or intrinsic dimensions $\left(I_{d}, V_{d}, V_{g}\right)$. To obtain the external or extrinsic characteristics $\left(I_{d s}\right.$, $V_{d s}, V_{q s}$ ) of the component, we have to take into consideration the effect of the parasitic access source resistance $R_{s}$, the drain resistance $R_{d}$ and also the effect of the resistance $R_{p}$ parallel to the channel on the polarization voltages values (Fig. 3).

To obtain the real expressions of the characteristics $I_{d s}\left(V_{d s}, V_{g s}\right)$, we have to substitute the intrinsic terms by the extrinsic terms in all the previous relations. Therefore

$V_{d}=V_{d s}+V_{l d}-\left(R_{s}+R_{d}\right) I_{d}$,

$V_{g}=V_{g s}+V_{l s}-R_{s} I_{d}$,
$I_{d}=I_{d s}-\left(V_{d} / R_{p}\right)$

\section{Results and discussion}

In order to validate the $I-V$ characteristics of the GaAs MESFET set up in the previous work, a simulation software based on different formulas and equations is exposed, as well as the obtained results and their discussions.

The numerical calculation of the drain current as a function of the polarization voltage calls the expressions (16) - (21) previously established.

The study has been carried out on two MESFET 1 and MESFET 2 [6] parameters of which are summarized in the following Table 1 . To calculate the voltages $V_{l d}$ and $V_{l s}$ (expressions (23a) and (23b)), the values of the used parameters $a_{1}, b_{1}, c_{1}$, and $V_{l} / V_{p}$ are tabulated in the Table 2.

In order to check the validity of our model, we have compared the theoretical results with the experimental ones for the MESFET 1 and MESFET 2.

In Figs $4 a$ and $b$, we have respectively represented the comparison of the measured $I_{d s}\left(V_{d s}, V_{g s}\right)$ characteristics and the calculated ones by the simulation for the MESFET 1 and MESFET 2. In the linear regime, i.e., at a weak drain voltage polarization, we notice a good agreement between the experimental values and the simulation ones for both transistors. When the drain voltage increases and becomes more important, we notice a certain difference between the experimental values and the results of the simulation. This difference progressively increases until the saturation. This difference is mainly caused by the approximations made in the mathematical model and the simulation software, it also stems from the geometric parameters effects and existence of parasitic quantum phenomenon, which we have not taken into consideration. In the saturation regime, when the drain voltage gets important, we notice that the theoretical results are in a good agreement with experimental ones. In conclusion, we also remark that the theoretical and the experimental results have the similar behavior towards the drain voltage and coincide well, notably at high values of the $V_{d s}$ voltage. This shows that the method is well founded.

\section{- Effect of source and drain parasitic resistances}

In order to put into evidence the effects of the source and drain parasitic resistances $R_{S}$ and $R_{d}$ on the $I$ - $V$ characteristics of the GaAs MESFET, in Figs 5 a and $\mathrm{b}$, in the case of the previous two transistors, we present the variations of the drain current as a function of the drain voltage with and without the parasitic resistance.

\section{- $\quad$ Effect of the voltage $V_{l}(x, y)$}

When solving the two-dimensional Poisson equation, it should be taken into consideration two voltages existing at the sides of the conducting channel: $V_{l s}$ source side and $V_{l d}$ drain side. Despite their very 
weak values, these voltages influence the $I-V$ static transistor characteristics. In Figs $6 a$ and b, we present the effect of these side voltages for two studied structures.

\section{Conclusion}

In this paper, we have proposed an analytical study of the $I-V$ characteristics of the GaAs MESFET. The influence of parasitic elements and sides voltages $V_{l s}$ and $V_{l d}$ on the drain current $I_{d s}$ expression has been established, these latest voltages resulted from the two-dimensional analysis of the Poisson equation by the Green technique. This study allowed us to carry out a synthetic approach to this two-dimensional analysis to realize a valid exactness of the analytical model for static characteristics of the GaAs MESFET composant.

\section{References}

1. S. Khemissi, Master thesis // Faculty of Sciences, Constantine University (2003).

2. N. Merabtine, Ph.D thesis // Faculty of Engineering, Constantine University (2003).

3. M. Zaabat. Ph.D thesis // Faculty of Technology, Oum El Bouaghi University (2004).

4. J. Haslett et al. // IEEE Trans. Electron. Devices 47 (5) (2000).

5. H. Tran et al. // Ibid. 39 (9) (1992).

6. B. Janiguez et al. // Ibid. 46 (8) (1999).

7. S.P. Chin, C.Y. We // Ibid. 40 (4) (1993).

8. K.M. Shin, D.P. Klamer, J.I. Lion // Solid State Electronics 35 (11) (1992).

9. C.S. Chang, D.Y. Day // IEEE Trans. Electron. Devices 36 (2) (1989).

10. S.P. Murray, K.P. Roenker // Solid State Electronics 46 (2002). 